\title{
Application of mass-sensitive sensors - from molecules to cells
}

\author{
Franz L. Dickert \\ Department of Analytical Chemistry, University of Vienna, Waehringer Str. 38, 1090 Vienna, Austria \\ Franz.Dickert@univie.ac.at
}

\begin{abstract}
:
Mass-sensitive transducers are universally applicable since all absorbed or adsorbed analytes give a response. Preferred devices are QCMs, SAWs and in the last years e.g. FBARS. These transducers can be combined with sensitive and selective coatings. In this way analytes can be detected ranging from sub $\mathrm{nm}$ to some $\mu \mathrm{m}$ dimensions. Thus, both molecules and large biological particles are accessible. Favorable coatings can be synthesized by molecular imprinting. Bulk effects are used for molecules whereas surface patterning is preferred for proteins, viruses and cells.
\end{abstract}

Key words: QCMs, SAWs, Imprinting, Viruses, Cells

\section{Devices}

The importance of sensors in environmental monitoring, process control and medicine continuously increase to get analytical data. The reason for this fact is an improved miniaturizing of transducers and progress in receptor developing. An appreciable number of transducers with different sensitivities and fields of application are available. Only mass sensitive devices, however, will give a response to each analyte.

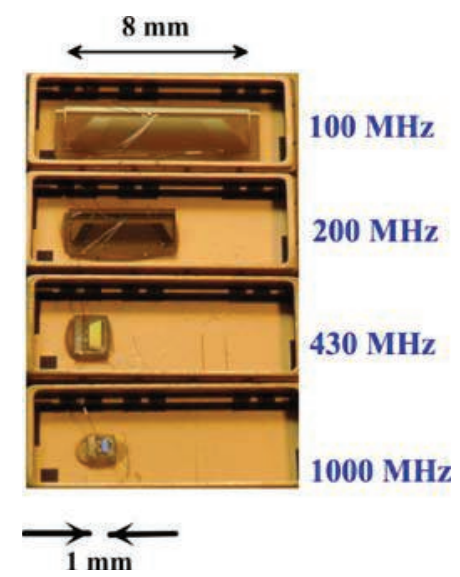

Fig. 1. SAWs with different frequencies from EPCOS

Furthermore, this allows label-free measurements. Figure 1 shows the progress in SAWs (Surface Wave Acoustic) devices in going to higher operating frequencies [1]. These acoustic resonators generate frequencies in dependence of electrode distances. The frequencies vary from typical $10 \mathrm{MHz}$ (QCM:
Quartz Crystal Microbalance), SAWs $1 \mathrm{GHz}$ and FBARs (thin-film bulk acoustic resonator) up to $10 \mathrm{GHz}$. The sensor responses increase in a quadratic manner in parallel to enhancing frequency.

\section{Sensitive Layers - Analytes}

The selective analyte detection is possible by combining these universal transducers with receptor layers designed by host-guest chemistry (Fig. 2) or molecular imprinting (Fig. $3)$.

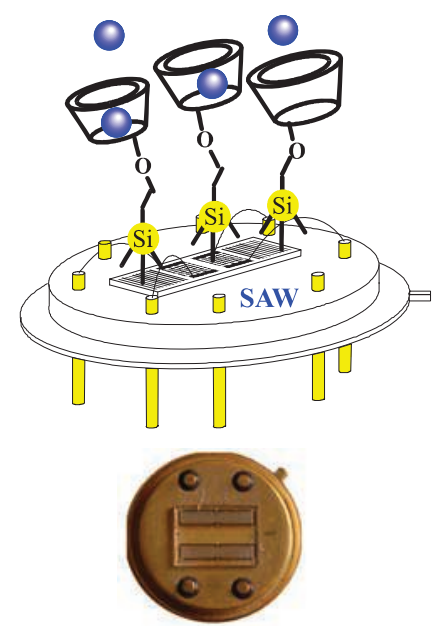

Fig. 2. SAWs and host-guest chemistry

Lean molecules can be selectively incorporated by molecular cavities. Paracyclophanes, cyclodexdrins and calixarines are suitable for this purpose since their cavity size and shape can be adapted to the analyte by synthetic 
efforts. Furthermore, these molecular hollows can be covalently linked to polymers. Then, these coating can be protected against destruction by e.g. flooding. The synthesis of tailored cavities is a time-consuming task, however.

A straightforward strategy is based on molecular imprinting to combine the molecular recognition capability of cavities with the robustness of polymers. In this case the coating can be generated directly on the transducers by polymerizing monomers around the analyte of interest. Thus, suitable receptors are generated for re-inclusion of the analyte. In Fig. 3 this is shown for adhered yeast cells on a QCM with imprinted polyurethane layer.

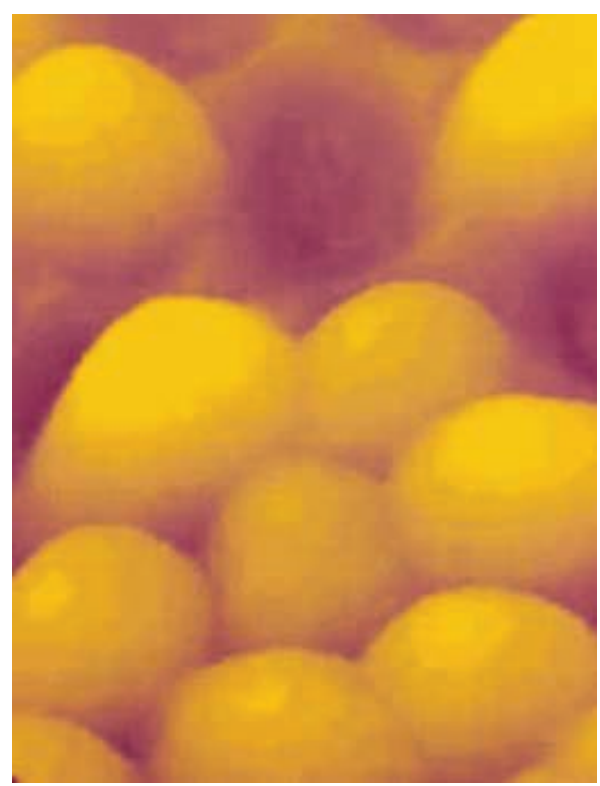

Fig. 3. Yeast cells imprinted polyurethane

In this way e.g. lean molecules, hormones, viruses and cells show responses as was shown in reference [2]. Thus, molecular cavities can be generated by biomimetic strategies to collect analytes in a size from nano-meters to some micro-meters. Lean molecules can be detected but also hormones such as estradiols and its derivatives. Proteins are of high biological importance. Especially, insulin can be enriched on coatings with mass-sensitive devices. This is possible with molecular imprinted polymers (MIPs) which are robust and insoluble materials. Human insulin and retard insulin can be distinguished which differ only by three amino acids. Many types of viruses are accessible to this measurement methods such as Foot and Mouth, Rhino, Tobacco Mosaic and Pox viruses. The particle sizes range from $30 \mathrm{~nm}$ to about $300 \mathrm{~nm}$. The more complex Pox virus has even a membrane. The imprinted polymers engulf nearly the whole virus. Thus, the viruses are characterized by their sizes, shapes and surface properties and not only by distinct interactions centres as in the case of natural antibodies.

These strategies can also be used for detection of different types of bacteria and yeast cells. Further developments allow to characterize e.g. blood cells which have a diameter of approximately $7 \mu \mathrm{m}$ [3]. In this case, the pronounced characteristic recognition step is the interaction of receptor surface with the erythrocyte membrane since these cells have a flexible shape. Thus, blood groups can be determined in this way.

\section{Conclusions}

Mass-sensitive transducers show the potential to detect nearly all types of analytes, both in gaseous and liquid phase. Especially. imprinting allows a straightforward adaption of the coating to the transducer. In going from QCM with typical $10 \mathrm{MHz}$ resonance frequency to SAWs appreciable gain in sensitivity can be obtained. Future developments will show potentials of high frequency FABRs. They show manifold applications in mobile phones as filters. Future perspectives will be in sensor science.

\section{References}

[1] A. Mujahid, F.L. Dickert, Surface Acoustic Wave (SAW) for Chemical Sensing - Applications of Recognition Layers, Sensors 17, 2716-2742 (2017); doi:10.3390/s17122716

[2] U. Latif, J. Qian, S. Can, F.L. Dickert, Biomimetic receptors for Bioanalyte Detection by Quartz Crystal Microbalances - From Molecules to Cells, Sensors 14, $23419-23438$ (2014); doi:10.3390/s141223419

[3] A. Mujahid, S. Aigner, F.L. Dickert, Microstructured interdigital capacitors with synthetic antibody receptors for $\mathrm{ABO}$ blood-group typing, Sensors and Actuators B: Chemical 242, 378383 (2017); doi.org/10.1016/j.snb.2016.11.056 